\title{
ОСОБЕННОСТИ УПРАВЛЕНИЯ ИЗМЕНЕНИЯМИ
} В МЕДИЦИНСКОЙ ОРГАНИЗАЦИИ В РАМКАХ РЕАЛИЗАЦИИ ФЕДЕРАЛЬНОГОПРОЕКТА ॥СОЗДАНИЕ НОВОЙ МОДЕЛИ МЕДИЦИНСКОЙ ОРГАНИЗАЦИИ, ОКАЗЫВАЮЩЕЙ \section{ПЕРВИЧНУЮ МЕДИКО-САНИТАРНУЮ ПОМОЩЬ"}

\author{
С. Н. Алексеенко ${ }^{1}$, В. Ф. Арженцов ${ }^{2}$, А. В. Верменникова ${ }^{1}$, А. В. Веселова ${ }^{1, *}$, \\ В. С. Аегтярев ${ }^{3}$, В. И. Стародубов ${ }^{4}$
}

${ }_{1}$ Федеральное государственное бюджетное образовательное учреждение высшего образования «Кубанский государственный медицинский университет» Министерства здравоохранения Российской Федерации, ул. им. Митрофана Седина, д. 4, г. Краснодар, 350063, Россия

${ }^{2}$ АО «Производственная система "Росатом"», пер. Гамсоновский д. 5, стр.3, г. Москва, 115191, Россия

${ }^{3}$ Государственное бюджетное учреждение здравоохранения «Городская больница г. Горячий Ключ» Министерства здравоохранения Краснодарского края, ул. Жемчужная, д. 35, г. Горячий Ключ, Краснодарский край, 353290, Россия

${ }^{4}$ Федеральное государственное бюджетное учреждение «Центральный научно-исследовательский институт организации и информатизации здравоохранения» Министерства здравоохранения Российской Федерации,

ул. Добролюбова, д. 11, г. Москва, 127254, Россия

\begin{abstract}
Аннотация
Цель: изучение причин сопротивления изменениям у сотрудников медицинской организации в рамках реализации федерального проекта «Создание новой модели медицинской организации, оказывающей первичную медико-санитарную помощь» и разработка рекомендаций по управлению изменениями на основе использования теории Э. Голдратта.
\end{abstract}

Материалы и методы. Необходимость изменений в организации процессов в лечебных учреждениях обусловлена реализацией приоритетного федерального проекта «Создание новой модели медицинской организации, оказывающей первичную медико-санитарную помощь», разработанного Министерством здравоохранения Российской Федерации при методической поддержке Производственной системы «Росатом». В основу новой модели медицинской организации положены непрерывные улучшения (кайдзен) с применением инструментов бережливого производства. Данная особенность требует иного подхода к управлению изменениями и преодолению сопротивления изменениям у медицинских работников. Исследовательскую выборку составили сотрудники ГБУЗ «Городская больница г. Горячий Ключ» Минздрава Краснодарского края. Метод исследования - анонимное анкетирование, в основе которого лежит теория Э. Голдратта.

Результаты. Предложенные в работе основные рекомендации по управлению изменениями в процессе перехода к новой модели медицинской организации позволяют руководителю учреждения здравоохранения комплексно оценить готовность его сотруд- 
ников к изменениям, а также осуществлять эффективную поэтапную трансформацию существующей модели медицинской организации.

Заключение. Данные рекомендации по управлению изменениями в процессе перехода к новой модели медицинской организации могут быть использованы руководителями любых медицинских учреждений для эффективного перехода организации на непрерывную оптимизацию процессов с применением инструментов бережливого производства с целью достижения критериев «новой модели».

Ключевые слова: новая модель медицинской организации, управление имениями, оптимизация процессов, сопротивление изменениям

Конфликт интересов: авторы заявили об отсутствии конфликта интересов.

Для цитирования: Алексеенко С.Н., Арженцов В.Ф., Верменникова Л.В., Веселова Д.В., Дегтярев В.С., Стародубов В.И. Особенности управления изменениями в медицинской организации в рамках реализации федерального проекта «Создание новой модели медицинской организации, оказывающей первичную медико-санитарную помощь». Кубанский научный медицинский вестник. 2019; 26(5): 18-28. https://doi.org/10.25207/16086228-2019-26-5-18-28

Поступила 12.08.2019

Принята после доработки 20.08.2019

Опубликована 25.10.2019

\title{
CHANGE MANAGEMENT IN A MEDICAL ORGANISATION DURING THE IMPLEMENTATION OF THE FEDERAL PROJECT "CREATION OF A NEW MODEL OF A MEDICAL ORGANISATION PROVIDING PRIMARY HEALTH CARE"
}

\author{
Sergey N. Alekseenko ${ }^{1}$, Vladimir F. Arzhentsov ${ }^{2}$, Lyudmila V. Vermennikova ${ }^{1}$, \\ Darya V. Veselova1,*, Victor S. Degtyarev ${ }^{3}$, Vladimir I. Starodubov ${ }^{4}$ \\ ${ }^{1}$ Kuban State Medical University, Ministry of Healthcare of the Russian Federation, \\ Mitrofana Sedina str., 4, Krasnodar, 350063, Russia \\ ${ }^{2}$ Rosatom Production System, \\ Gamsonovsky lane, 5, bld. 3, Moscow, 115191, Russia \\ ${ }^{3}$ Central City Hospital, Goryachy Klyuch, Ministry of Healthcare of the Russian Federation, \\ Zhemchuzhnaya str., 35, Goryachy Klyuch, Krasnodar Territory, 353290, Russia \\ ${ }^{4}$ Research Institute for Health Organization and Informatics, \\ Ministry of Health of the Russian Federation, \\ Dobrolyubova str., 11, Moscow, 127254, Russia
}

\begin{abstract}
Aim. To study reasons behind the resistance of medical workers to changes while implementing the federal project "Creation of a New Model of a Medical Organisation Providing Primary Health Care", as well as to develop guidelines on change management on the basis of Goldratt's theory.
\end{abstract}

Materials and methods. Changes in the process organisation of medical institutions are necessitated by the implementation of the federal project "Creation of a New Model of a Medical Organisation Providing Primary Health Care" developed by the Ministry of Healthcare of the 
Russian Federation with the methodological support of the Rosatom Production System. A new model of a medical organisation is based on continuous improvement (kaizen) using lean tools, which requires a different approach to managing changes and overcoming the resistance of medical workers to their implementation. The study sample included the employees of the Central City Hospital in Goryachy Klyuch (Krasnodar Territory). The research took the form of an anonymous questionnaire survey drawing on Goldratt's theory.

Results. Basic recommendations on the management of changes associated with the transitioning to the new model suggested in this work allow heads of healthcare institutions to evaluate employees' readiness to changes comprehensively, as well as to transform the existing model of a medical organisation effectively and gradually.

Conclusion. These guidelines on change management when transitioning to the new model may be used by heads of medical institutions in order for the organisation to achieve effective transition to the continuous process optimisation with the use of lean tools, aiming to meet the criteria of the new model.

Keywords: new medical organisation model, change management, process optimisation, resistance to changes

Conflict of interest: the authors declare no conflict of interest.

For citation: Alekseenko S.N., Arzhentsov V.F., Vermennikova L.V., Veselova D.V., Degtyarev V.S., Starodubov V.I. Change Management in a Medical Organisation during the Implementation of the Federal Project "Creation of a New Model of a Medical Organisation Providing Primary Health Care". Kubanskii Nauchnyi Meditsinskii Vestnik. 2019; 26(5): 18-28. (In Russ., English abstract). https://doi.org/10.25207/1608-6228-2019-26-5-18-28

Submitted 12.08.2019

Revised 20.08.2019

Published 25.10.2019

\section{Введение}

Одним из ключевых направлений «Программы развития здравоохранения РФ», утвержденной в 2017 году, стал приоритетный федеральный проект «Создание новой модели медицинской организации, оказывающей первичную медикосанитарную помощь». Целью данного проекта является повышение удовлетворенности населения качеством оказания медицинской помощи. Его реализация обеспечивается путем применения философиии, принципов и инструментов бережливого производства в организации работы лечебно-профрилактических учреждений [1-4].

Бережливое производство - это концепция управления, основанная на устранении всех видов потерь путем формирования непрерывного потока создания ценности с охватом всех процессов организации и их постоянного совершенствования через вовлечение персонала в проведение улучшений [5].

В основе данной концепции лежит идея непрерывных улучшений (кайдзен) или изменений к лучшему. И в этой связи важной задачей медицинских организаций в реализации федерального проекта помимо освоения философии, принципов и инструментов бережливого производства становится управление изменениями.
Об этом свидетельствуют критерии, обозначенные в фредеральном проекте, описывающие целевое состояние современных организаций сферы здравоохранения. Двадцать один критерий сгруппирован по девяти основным блокам, среди которых: управление потоками пациентов, качество пространства, управление запасами, стандартизация процессов, качество медицинской помощи, доступность медицинской помощи, вовлеченность персонала в улучшения процессов, формирование системы управления, эффективность использования оборудования [5].

Помимо критериев, описывающих конечное состояние системы, как, например, количество пересечений потоков при проведении диспансеризации, профрилактических медицинских осмотров с иными потоками пациентов, есть критерии, которые отражают такую характеристику медицинской организации, как гибкость и адаптивность, то есть способность постоянно изменяться с целью повышения качества оказываемой медицинской помощи и роста удовлетворенности пациентов. К таким критериям можно отнести: пересмотр стандартов улучшенных процессов (не реже 1 раза в год), вовлеченность руководителей медицинских организаций и их заместителей во внедрение бережливых технологий (не менее 1 проекта в год у каждого 
руководителя или его заместителя), существование системы подачи и реализации предложений по улучшению.

В этой связи решение каждой задачи по управлению изменениями в процессе создания новой модели медицинской организации будет иметь свои особенности, а именно: анализ и совершенствование процессов должны обязательно проводиться с вовлечением максимального числа работников медицинской организации. Для этого необходимо использовать листы проблем и предложений, а также иные методики (анкетирование, метод фокус-групп и т.д.), позволяющие сотрудникам высказать свое мнение относительно направлений будущих изменений. Руководитель не должен единолично принимать решения о внедрении мероприятий по оптимизации работы, так как это приведет к сопротивлению изменениям со стороны сотрудников. Согласно принципам бережливого производства решения принимаются коллегиально с участием членов (проектных) рабочих групп [6] и с учетом мнения пациентов, для этого проводится системная работа по сбору проблем и предложений.

Преодоление сопротивления изменениям является самой важной задачей в процессе перехода к новой модели медицинской организации, оказывающей первичную медико-санитарную помощь. Под сопротивлением персонала изменениям системы управления принято понимать негативную реакцию системы, групп и отдельных лиц, затрудняющую процесс проведения изменений, угрожающую сложившейся культуре организации и структуре управления. Первая реакция на изменения - это сопротивление, так как работникам требуется время, чтобы оценить издержки и выгоды перемен для себя [7, 8].

Преодоление сопротивления изменениям является одним из важнейших факторов, определяющих эффрективность изменений в каждом конкретном случае, поскольку внедрение технологии бережливого производства требует не только и не столько освоения новых способов выполнения работы, сколько овладение инструментами анализа способов решения проблем. Только благодаря правильному и глубокому анализу возможно, выявить коренную причину и определить верный алгоритм необходимых действий с целью решения проблемы.

Резюмируя вышеизложенное, стоит отметить, что, в отличие от большинства изменений в организациях, имеющих временный характер (реорганизация, адаптация к изменениям законодательства и т.д.), внедрение технологий бережливого производства в сфрере здравоохранения призвано сформировать культуру постоянных изменений. Поэтому залогом эффективности управления изменениями в процессе создания новой модели медицинской организации, оказывающей первичную медико-санитарную помощь, является вовлечение персонала, формирование бережливого мышления и культуры постоянных улучшений $[9,10]$.

Цель работы: изучение причин сопротивления изменениям у сотрудников медицинской организации, в рамках реализации федерального проекта «Создание новой модели медицинской организации, оказывающей первичную медикосанитарную помощь» и разработка рекомендаций по управлению изменениями на основе использования теории Э. Голдратта.

\section{Материалы и методы}

Проведено эмпирическое исследование, целью которого стала апробация теории Э. Голдратта для выявления отношения работников медицинской организации к изменениям, реализуемым в процессе перехода к новой модели. Результаты исследования легли в основу рекомендаций по корректировке стратегии управления изменениями (рис. 1) [7].

Исследовательскую выборку составили 85 сотрудников ГБУЗ «Городская больница г. Горячий Ключ» Минздрава Краснодарского края, из них 58\% врачей, 33\% средних медицинских работников и 9\% младшего медицинского персонала. Данная медицинская организация участвует в реализации проекта «Создание новой модели медицинской организации, оказывающей первичную медико-санитарную помощь» со 2-го квартала 2018 года.

Специалистами ФГБО ВО «Кубанский государственный медицинский университет» Минздрава России было проведено анонимное анкетирование, основанное на теории Э. Голдратта, согласно которому респонденту предлагалось в свободной форме сорормулировать 4 группы утверждений, касающихся внедрения бережливого производства в медицинские организации: преимущества

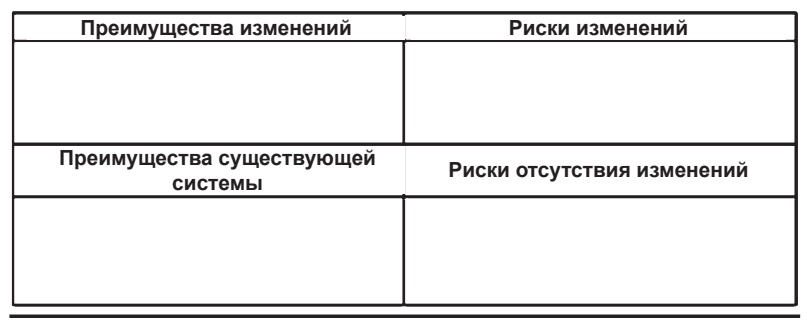

Puc. 1. Матрица сопротивления переменам.

Fig. 1. Resistance-to-change matrix. 
изменений, риски изменений, преимущества существующей системы и риски отсутствия изменений. Данная методика позволяет руководителю учреждения здравоохранения комплексно оценить готовность его сотрудников к изменениям и за счет этого осуществлять эффрективную поэтапную трансформацию существующей модели медицинской организации. Для статистической обработки полученной информации применяли пакет «Анализ данных» программы Microsoft Office Excel-2010. Статистически значимую достоверность различий долей принимали при $\mathrm{p}<$ 0,05 по критерию хи-квадрат.

\section{Результаты и обсуждение}

Данные из первой группы «преимущества изменений» используются с целью выявления ценности изменений для сотрудников медицинских организаций. В дальнейшем эта информация должна использоваться для мотивации сотрудников.

По результатам проведенного исследования около половины респондентов позитивной направленностью внедрения бережливого производства считают оптимизацию и стандартизацию процессов (47\%). Вторым по количеству упоминаний стал фрактор сокращения времени протекания процессов (30\%). Ценность цифровизации как результата внедрения бережливых технологий отметили лишь 9\% опрошенных и только 5\% считали, что изменения приведут к сокращению затрат (рис. 2)

Таким образом, с целью преодоления сопротивления изменениям руководителю данной организации необходимо реализовывать краткосрочные проекты с видимыми результатами. Важно создать «прозрачную» систему мониторинга и визуализации ключевых показателей проектов, доступную для каждого сотрудника и пациента медицинской организации, так как зачастую информацией о ходе реализации проектов обладают только члены рабочей группы и руководитель, что препятствует росту вовле-

\section{Преимущества изменений}

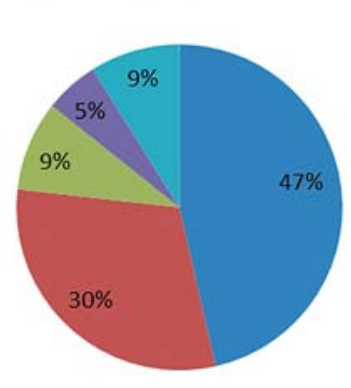

Оптимизация и
стандартизация процессов
работы
Сокращение времени
протекания процессов
Шифровизация
Ц Снижение затрат
$=$ Прочее

Рис. 2. Преимущества изменений.

Fig. 2. Benefits of change. ченности в работу по поиску и устранению потерь остальных членов коллектива.

Кроме того, респондентам было предложено указать, какие, по их мнению, существуют риски для их медучреждения в случае, если изменения не будут реализованы (рис. 3). Наибольший вес набрал фактор снижения качества медицинской помощи. Однако 31\% работников не видят никаких угроз в сохранении текущего положения.

Интерпретацией данной позиции можно считать отсутствие в сознании работников необходимости менять что-либо в целом. В этой связи руководителю необходимо формировать убеждение о необходимости и целесообразности перемен, поскольку данный фактор является залогом конкурентоспособности и эффрективности любых организационных преобразований, необходимо формировать уверенность в возможности конкурировать с частными медицинскими организациями.

Согласно теории изменений К. Левина первым шагом на пути к переменам является отказ от привычных способов действий, основанный на убежденности в их неэффективности, невозможности дальнейшего использования [11]. Без осознания сотрудниками необходимости изменений невозможно перейти на следующую стадию. Обучение концепции «бережливое производство» в таком случае будет неэффективным, новые способы выполнения работы не будут восприняты и вызовут только раздражение и непонимание. Именно поэтому важным является качественное «размораживание», в терминологии К. Левина, старых установок медицинских работников. Необходимо проведение анализа текущей ситуации, в том числе с помощью опроса пациентов и сотрудников, определения направления и объекта необходимых изменений; разработка единого видения цели и ее трансляция, составление плана изменений, личный пример руководителя и его заместителей.

\section{Риски отсутствия изменений}

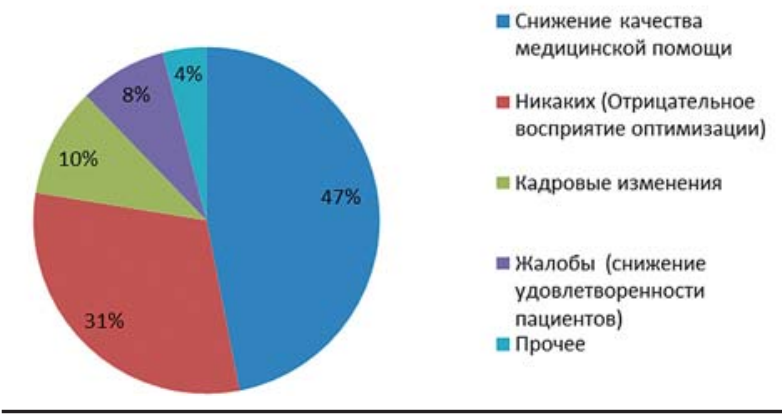

Рис. 3. Риски отсутствия изменений.

Fig. 3. Risks of no change. 
Анализ ответов, отраженных в графрах «риски изменений» и «преимущества существующей системы», показал следующие результаты. Наибольшее количество респондентов высказали свои опасения насчет эффеектиности перемен. Работники утверждали, что изменения не будут доведены до конца (рис. 4), исходя из чего можно сделать заключение, что сотрудники данной районной больницы не в полной мере понимают суть философии бережливого производства. Для преодоления такого рода опасений в сознании персонала рекомендуется реализовать серию краткосрочных значимых проектов длительностью не более 3 месяцев с небольшими, но видимыми результатами улучшений, а также акцентировать внимание на роли каждого сотрудника в принятии решений при реализации проектов. Быстрый результат краткосрочных проектов будет являться наглядной демонстрацией эффективности от внедрения технологии «бережливого производства» в медицинскую организацию.

Также большое количество рисков связано с человеческим фактором: сложность адаптации (19\%), риски увеличения нагрузки на персонал (9\%), риск увольнения (9\%). Для преодоления сопротивления изменениям, вызванного вышеперечисленными факторами, необходимо оказывать содействие сотрудникам в получении новых навыков, оказывать помощь и поддержку для эффрективной адаптации в новой системе. С этой целью рекомендуется применение каскадной технологии обучения персонала. Ее суть состоит в том, что специалисты, прошедшие курс повышения квалификации по основам бережливого производства, передают накопленный опыт и знания, полученные в ходе обучения, сотрудникам, стоящим ниже на одну ступень «должностной лестницы», затем эти сотрудники передают полученный опыт звеньям ниже, и так пока не будет обучен

\section{Риски изменений}

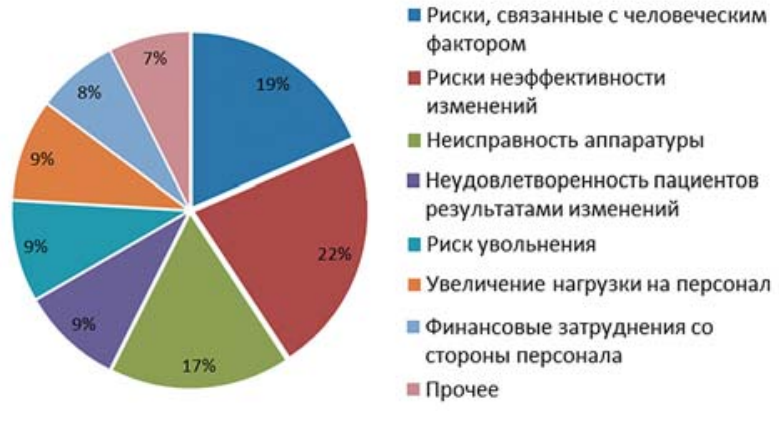

Pис. 4. Риски изменений.

Fig. 4. Risks associated with change. весь персонал. Важно обучить первичный пул сотрудников в специализированных учебных центрах для обеспечения высокого уровня качества полученных знаний и навыков, которые будут многократно воспроизводиться в организации. Обучение каскадным методом является наиболее простым и быстрым способом донесения информации до всех работников организации [12].

Возвращаясь к анализу рисков изменений, стоит отметить, что, с одной стороны, преимуществами изменений работники ГБУЗ «Городская больница г. Горячий Ключ» Минздрава Краснодарского края считают цифровизацию, однако, с другой, 17\% высказывает опасения насчет неисправности аппаратуры, которая может поставить под угрозу эффективность произведённых улучшений.

Блок утверждений, посвященных формулировке преимуществ существующей системы, призван идентифицировать ценные для сотрудников характеристики организации, сотрудниками которой они являются. В дальнейшем при реализации изменений необходимо декларирование намерения сохранения данных преимуществ и выполнение данного обещания.

Как следует из рисунка 5, для сотрудников данной медицинской организации главным преимуществом в условиях работы является коллектив и руководство (39\%). Также в списке преимуществ оказались стабильность (25\%), существующий график работы (22\%).

На наш взгляд, полученные результаты исследования являются довольно стандартными для организаций сферы здравоохранения, участвующих в реализации приоритетного федерального проекта «Создание новой модели медицинской организации, оказывающей первичную медико-санитарную помощь».

\section{Преимущества существующей системы}

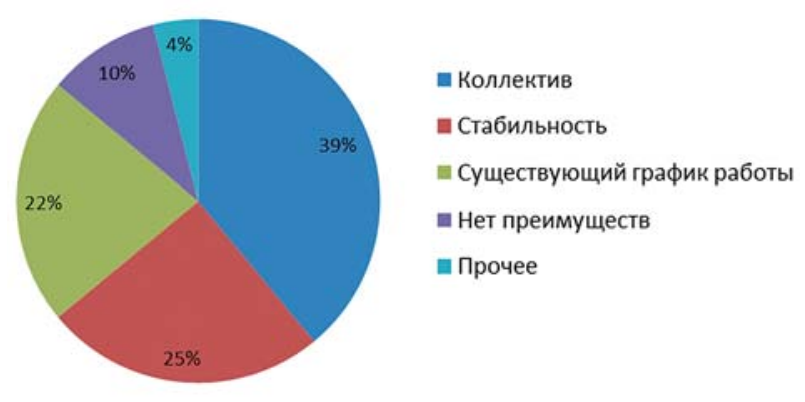

Pис. 5. Преимущества существующей системы. Fig. 5. Advantages of the existing system. 


\section{Заключение}

На основании проведенного анализа, в основе которого лежит теория Э. Голдратта, были сформулированы рекомендации по управлению изменениями в представленной медицинской организации:

1) разработка единого видения цели изменений, декларирование главным врачом намерения сохранить те преимущества организации, которые важны для сотрудников;

2) назначение ответственного за внедрение из числа заместителей главного врача, поиск лидеров изменений среди всех сотрудников медицинской организации и вовлечение их в работу по улучшениям;

3) необходимо начинать с серии краткосрочных проектов по улучшениям, дающих видимый результат, направленных на оптимизацию процессов работы и сокращение времени протекания процессов, в том числе с личного проекта главного врача и его заместителей. Затем можно переходить к открытию долгосрочных проектов;

4) постоянное информирование работников о ходе реализации проектов и о достигнутых результатах первым лицом на открытых собраниях коллектива;

\section{Вклад авторов}

\section{Алексеенко С.Н.}

Разработка концепции - формирование идеи; формулировка и развитие ключевых целей и задач.

Проведение исследования - анализ и интерпретация полученных данных.

Подготовка и редактирование текста - составление черновика рукописи, его критический пересмотр с внесением ценного интеллектуального содержания; участие в научном дизайне.

Утверждение окончательного варианта - принятие ответственности за все аспекты работы, целостность всех частей статьи и ее окончательный вариант.

Ресурсное обеспечение исследования - предоставление вычислительной техники для анализа.

\section{Арженцов В.Ф.}

Разработка концепции - формирование идеи; фрормулировка и развитие ключевых целей и задач.

Проведение исследования - анализ и интерпретация полученных данных.

Подготовка и редактирование текста - составление черновика рукописи, его критический пересмотр с внесением ценного интеллектуального содержания; участие в научном дизайне.
5) организация обучения сотрудников философии, инструментам и методам бережливого производства по каскадной технологии;

6) создание системы мотивации, поддерживающей непрерывные улучшения, проведение широкой информационной кампании в ее поддержку. При ее разработке следует опираться на четыре группы фракторов теории Э. Голдратта.

Таким образом, вышеизложенные рекомендации могут быть использованы для преодоления сопротивления изменениям функционирования организации у работников в процессе перехода к новой модели медицинской организации, оказывающей первичную медико-санитарную помощь.

\section{Соответствие принципам этики}

Проведенное исследование соответствует стандартам Хельсинкской декларации (Declaration Helsinki). От всех анкетированных получено письменное добровольное информированное согласие на участие в исследовании.

\section{Compliance with the ethics principles}

The study complies with the standards set in the Helsinki Declaration. All examined patients gave their written voluntary informed consent to participate in the study.
Утверждение окончательного варианта - принятие ответственности за все аспекты работы, целостность всех частей статьи и ее окончательный вариант.

\section{Верменникова Л.В.}

Разработка концепции - фрормулировка ключевых целей и задач.

Проведение исследования - сбор и анализ данных.

Подготовка и редактирование текста - составление черновика рукописи, его критический пересмотр с внесением ценного интеллектуального содержания; участие в научном дизайне.

Утверждение окончательного варианта - принятие ответственности за все аспекты и целостность работы.

Подготовка и создание опубликованной работы в части визуализации и отображения данных.

Применение статистических и вычислительных методов для анализа и синтеза данных исследования.

\section{Веселова Д.В.}

Разработка концепции - формулировка ключевых целей и задач.

Проведение исследования - сбор и анализ данных.

Подготовка и редактирование текста - составление черновика рукописи, его критический пересмотр 
с внесением ценного интеллектуального содержания; участие в научном дизайне.

Утверждение окончательного варианта - принятие ответственности за все аспекты и целостность работы.

Применение статистических и вычислительных методов для анализа и синтеза данных исследования.

Подготовка и создание опубликованной работы в части визуализации и отображения данных.

\section{Дегтярев В.C.}

Разработка концепции - формулировка ключевых целей и задач.

Проведение исследования - сбор, анализ и интерпретация данных.

Подготовка и редактирование текста - составление черновика рукописи, его критический пересмотр с внесением ценного интеллектуального содержания; участие в научном дизайне.
Утверждение окончательного варианта - принятие ответственности за все аспекты и целостность работы.

Ресурсное обеспечение исследования - предоставление объектов и материалов исследования для анализа.

\section{Стародубов В.И.}

Разработка концепции - формирование идеи; фрормулировка и развитие ключевых целей и задач.

Проведение исследования - анализ и интерпретация полученных данных.

Подготовка и редактирование текста - составление черновика рукописи, его критический пересмотр с внесением ценного интеллектуального содержания; участие в научном дизайне.

Утверждение окончательного варианта - принятие ответственности за все аспекты работы, целостность всех частей статьи и ее окончательный вариант.

\section{Author contribution}

\section{S.N. Alekseenko}

Concept development - idea development; definition and development of key aims and objectives.

Conducting research - analysis and interpretation of the obtained data.

Manuscript preparation and editing - drafting of the manuscript, critical revision of the draft manuscript for important intellectual content; participation in scientific design.

Approval of the final manuscript - accountability for all aspects of the work, including the integrity of all parts of the article and its final version.

Resource support - provision of computer equipment for analysis.

\section{V.F. Arzhentsov}

Concept development - idea development; definition and development of key aims and objectives.

Conducting research - analysis and interpretation of the obtained data.

Manuscript preparation and editing - drafting of the manuscript, critical revision of the draft manuscript for important intellectual content; participation in scientific design.

Approval of the final manuscript - accountability for all aspects of the work, including the integrity of all parts of the article and its final version.

\section{L.V. Vermennikova}

Concept development — definition key aims and objectives.

Conducting research - data collection and analysis.

Manuscript preparation and editing - drafting of the manuscript, critical revision of the draft manuscript for important intellectual content; participation in scientific design.
Approval of the final manuscript - accountability for all aspects of the work, including the integrity of all parts of the article.

Preparation and creation of the published work in terms of visualisation and data display.

\section{D.V. Veselova}

Concept development — definition key aims and objectives.

Conducting research — data collection and analysis.

Manuscript preparation and editing - drafting of the manuscript, critical revision of the draft manuscript for important intellectual content; participation in scientific design.

Approval of the final manuscript - accountability for all aspects of the work, including the integrity of all parts of the article.

Analysis and synthesis of research data using statistical and computational methods.

Preparation and creation of the published work in terms of visualisation and data display.

\section{V.S. Degtyarev}

Concept development — definition key aims and objectives.

Conducting research — data collection, its analysis and interpretation.

Manuscript preparation and editing - drafting of the manuscript, critical revision of the draft manuscript for important intellectual content; participation in scientific design.

Approval of the final manuscript - accountability for all aspects of the work, including the integrity of all parts of the article.

Resource support - provision of subjects and materials for analysis. 


\section{V.I. Starodubov}

Concept development - idea development; definition and development of key aims and objectives.

Conducting research - analysis and interpretation of the obtained data.
Manuscript preparation and editing - drafting of the manuscript, critical revision of the draft manuscript for important intellectual content; participation in scientific design.

Approval of the final manuscript - accountability for all aspects of the work, including the integrity of all parts of the article and its final version.

\section{Список литературы}

1. Новая модель медицинской организации, оказывающей первичную медико-санитарную помощь. Методические рекомендации. Электронный фронд правовой и нормативно-технической документации; 2019. URL: http://docs. cntd.ru/document/560498624

2. Лайкер Д.К., Майер Д. Дао Toyota: 14 принципов менеджмента ведущей компании мира. Пер. с англ. 9-е изд. М.: Издательская группа «Точка»; 2017. 592 c.

3. Детмер У. Теория ограничений Голдратта: Cucтемный подход к непрерывному совершенствованию. 8-е изд. М.: Альпина Бизнес Букс; 2017. 444 с.

4. Логинова Т.В. Причины сопротивления организационным изменениям и пути их преодоления. Наука 21 века: вопросы, гипотезы, ответы. 2013; 1: 101-114.

5. Арженцов В.Ф. Применение принципов бережливого производства в медицине. Управление качеством в здравоохранении. 2018; 1: 14-19.

6. Арженцов В.Ф., Гайворонская Т.В., Веселова Д.В., Верменникова Л.В., Чабанец Е.А. Оценка эффективности метода «обучения действием» на «Фабрике процессов» с целью применения философии, принципов и инструментов бережливого производства в медицинских организациях. Современные проблемы науки и образования. 2019; 2. URL: http://www.science-education.ru/article/ view?id=28596. DOI: 10.17513/spno.28596

7. Галынчик Т.А. Управление изменениями: учебное пособие. Нижневартовск: Изд-во НВГУ; 2016. 120 с.
8. Боброва В.Е. Каскадное обучение как метод развития кадрового потенциала структурного подразделения ООО «РЖД». Решетневские чтения. 2017; 2: 549-550.

9. Паспорт проекта "Создание новой модели медицинской организации, оказывающей первичную медико-санитарную помощь». Министерство здравоохранения Российской Федерации; 2018 [обновлено 27.03.2018]. URL: https://www. rosminzdrav.ru/ministry/61/22/informatsionnyematerialy-po-napravleniyu-strategicheskogorazvitiya-rossiyskoy-federatsii-zdravoohranenie/ sozdanie-novoy-modeli-meditsinskoy-organizatsiiokazyvayuschey-pervichnuyu-mediko-sanitarnuyupomosch

10. Вергазова Э. Бережливая поликлиника: неожиданные проблемы и как их решить. Заместитель главного врача. 2018; 3: 14-25.

11. Казарин Б.В., Камушкина Л.В., Подубный В.Н., Ясько Б.А. Оценка эфффективности фрормирования управленческих компетенций врача-руководителя в процессе послевузовского образования. Международный журнал прикладных и ффундаментальных исследований. 2017; 4(1): 127-132.

12. Редько А.Н., Шильцова Т.А., Савчук С.Б. Основные социально-экономические показатели, характеризующие современное состояние системы здравоохранения Российской Федерации. Научный вестник Южного института менеджменma. 2018; 1: 52-56. DOI: 10.31775/2305-3100-2018$1-52-56$

\section{References}

1. Pasport proekta «Sozdanie novoi modeli meditsinskoi organizatsii, okazyvayushchei pervichnuyu mediko-sanitarnuyu pomoshch'» [Passport of the project "Creating a new model of a medical organization providing primary health care"]. Ministerstvo zdravookhraneniya Rossiiskoi Federatsii; 2018 [updated 27.03.2018]. Available mode: https://www.rosminzdrav.ru/ministry/61/22/informatsionnye-materialy-po-napravleniyu-strategicheskogo-razvitiya-rossiyskoy-federatsii-zdravoohranenie/ sozdanie-novoy-modeli-meditsinskoy-organizatsii-okazyvayuschey-pervichnuyu-mediko-sanitarnuyu-pomosch

2. Vergazova E. Berezhlivaya poliklinika: neozhidannye problemy i kak ikh reshit' [Lean Polyclinic: unexpected problems and how to solve them]. Zamestitel' Glavnogo Vracha. 2018; 3: 14-25. (In Russ.).

3. Kazarin B.V., Kamushkina L.V., Podubnyi V.N., Yas'ko B.A. Otsenka effektivnosti formirovaniya upravlencheskikh kompetentsii vracha-rukovoditelya v protsesse poslevuzovskogo obrazovaniya [Evaluation of the effectiveness of the formation of managerial competencies of the head doctor in the process of postgraduate education]. International Journal of Applied and Fundamental Research. 2017; 4(1): 127-132 (In Russ.).

4. Red'ko A.N., Shil'tsova T.A., Savchuk S.B. Main socio-economic indicators of the current state of the health of the Russian Federation. Nauchnyi Vestnik Yuzhnogo Instituta Menedzhmenta. 2018; 1: 52-56 (In Russ., English abstract). DOI: 10.31775/23053100-2018-1-52-56 
5. Novaya model' meditsinskoi organizatsii, okazyvayushchei pervichnuyu mediko-sanitarnuyu pomoshch' Metodicheskie rekomendatsii [A new model of a primary health care organization. Guidelines]. Elektronnyi fond pravovoi i normativno-tekhnicheskoi dokumentatsii; 2019 (In Russ.). Available mode: http:// docs.cntd.ru/document/560498624

6. Laiker D.K., Maier D. Dao Toyota: 14 printsipov menedzhmenta vedushchei kompanii mira [Dao Toyota: 14 principles of management of a leading company in the world]. Transl. from Engl. 9th ed. Moscow: Izdatel'skaya gruppa "Tochka”; 2017. 592 p. (In Russ.).

7. Detmer U. Teoriya ogranichenii Goldratta: Sistemnyi podkhod k nepreryvnomu sovershenstvovaniyu [Goldratt's Theory of Constraints: A Systems Approach to Continuous Improvement]. 8th ed. Moscow: Al'pina Biznes Buks; 2017. 444 p. (In Russ.).

8. Loginova T.V. Prichiny soprotivleniya organizatsionnym izmeneniyam i puti ikh preodoleniya [Reasons for resistance to organizational changes and ways to overcome them]. Nauka 21 veka: Voprosy, Gipotezy, Otvety. 2013; 1: 101-114 (In Russ.).
9. Arzhentsov V.F. Primenenie printsipov berezhlivogo proizvodstva $v$ meditsine [Application of lean manufacturing principles in medicine]. Upravlenie Kachestvom $v$ Zdravookhranenii. 2018; 1: 14-19 (In Russ.).

10. Arzhentsov V.F., Gaivoronskaya T.V., Veselova D.V., Vermennikova L.V., Chabanets E.A. Evaluation of the efficiency of the method of "learning by action" at the "factory of processes" for the purpose of application of philosophy, principles and instruments of protective production in medical organizations. Sovremennye Problemy Nauki i Obrazovaniya. 2019; 2 (In Russ., English abstract). URL: http://www.science-education. ru/article/view?id=28596. DOI: 10.17513/spno.28596

11. Galynchik T.A. Upravlenie izmeneniyami: uchebnoe posobie [Change Management: A Tutorial]. Nizhnevartovsk: Izd-vo NVGU; 2016. 120 p. (In Russ.).

12. Bobrova V.E. Cascade training as a method of development of personnel potential of the structural unit of OOO "Russian railways". Reshetnevskie Chteniya. 2017; 2: 549-550 (In Russ., English abstract).

\section{Сведения об авторах / Information about the authors}

Алексеенко Сергей Николаевич - доктор медицинских наук, доцент, ректор, заведующий кафедрой профилактики заболеваний, здорового образа жизни и эпидемиологии федерального государственного бюджетного образовательного учреждения высшего образования «Кубанский государственный медицинский университет» Министерства здравоохранения Российской Федерации.

Арженцов Владимир Федорович - эксперт АО «Производственная система "Росатом"».

Верменникова Людмила Викторовна - кандидат экономических наук, доцент кафедры общественного здоровья и здравоохранения ФПК и ППС федерального государственного бюджетного образовательного учреждения высшего образования «Кубанский государственный медицинский университет» Министерства здравоохранения Российской Федерации.

Веселова Дарья Валерьевна* - ассистент кафедры общественного здоровья здравоохранения и истории медицины федерального государственного бюджетного образовательного учреждения высшего образования «Кубанский государственный медицинский университет» Министерства здравоохранения Российской Федерации.

Контактная информация: e-mail: VeselovaDV@ ksma.ru, тел.: +7 (918) 957-06-70;

ул. им. Митрофрана Седина, д. 4, г. Краснодар, 350063, Россия.
Sergey N. Alekseenko - Dr. Sci. (Med.), Assoc. Prof., Rector, Head of the Department of Disease Prevention, Healthy Lifestyles and Epidemiology, Kuban State Medical University.

Vladimir F. Arzhentsov - Expert, Rosatom, JSC.

Lyudmila V. Vermennikova - Cand. Sci. (Economics), Assoc. Prof., Department of Public Health and Healthcare, Faculty of Advanced Training, Kuban State Medical University.

Daria V. Veselova * - Research Assistant, Departament of Public Health and the History of Medicine, Kuban State Medical University.

Contact information: email: VeselovaDV@ksma.ru, tel.: +7 (918) 957-06-70;

Mitrofana Sedina str., 4, Krasnodar, 350063, Russia. 
Дегтярев Виктор Сергеевич - кандидат медицинских наук, главный врач государственного бюджетного учреждения здравоохранения «Городская больница г. Горячий Ключ» Министерства здравоохранения Краснодарского края.

Стародубов Владимир Иванович - доктор медицинских наук, профессор, академик $\mathrm{PAH}$, директор федерального государственного бюджетного учреждения «Центральный научно-исследовательский институт организации и информатизации здравоохранения» Министерства здравоохранения Российской Федерации.

* Автор, ответственный за переписку / Corresponding author
Victor S. Degtyarev - Cand. Sci. (Med.), Chief Physician, Goryachiy Klyuch City Hospital, Krasnodar Krai.

Vladimir I. Starodubov - Dr. Sci. (Med.), Prof., RAS Academician, Director of the Central Scientific Research Institute of Organization and Informatization of Healthcare. 